\title{
First observation of ion beam channeling in bent crystals at multi-TeV energies
}

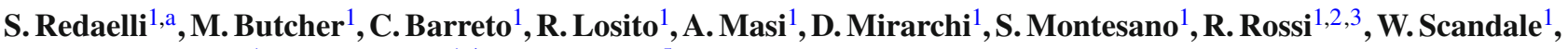 \\ P. Serrano Galvez ${ }^{1}$, G. Valentino ${ }^{1,4}$, F. Galluccio ${ }^{5}$ \\ ${ }^{1}$ European Organization for Nuclear Research CERN, Geneva, Switzerland \\ 2 INFN Sezione di Roma, Piazzale Aldo Moro 2, 00185 Rome, Italy \\ ${ }^{3}$ Dipartimento di Fisica, Sapienza Univ. Roma, Piazzale A.Moro, 2, 00185 Rome, Italy \\ ${ }^{4}$ University of Malta, Msida MSD2080, Malta \\ ${ }^{5}$ INFN Sezione di Napoli, Complesso Universitario di Monte Sant'Angelo, Via Cintia, 80126 Naples, Italy
}

Received: 15 November 2018 / Accepted: 27 January 2021 / Published online: 9 February 2021

(C) The Author(s) 2021

\begin{abstract}
Planar channeling in bent crystals has been observed in LHC with multi-TeV proton beam in 2015. Two crystals, mounted on novel high-accuracy goniometers (one in the horizontal and one in the vertical plane), are integrated in the LHC collimation system, for studying the feasibility of the crystal-based collimation scheme. Using this experimental setup, tests with fully-stripped lead ion beams at both $450 \mathrm{Z}$ and $6500 \mathrm{Z} \mathrm{GeV}$ were carried during dedicated LHC beam time. Planar channeling was observed for the first time with lead ions at these unprecedented energies surpassing by more than 1 order of magnitude the previous state-of-the-art for lead heavy ions and providing an important experimental basis for future applications of bent crystals in beam manipulations. The set of measurements performed to confirm this observation, as the local loss reduction in presence of channeling and the evidence of a deflected beam downstream of the crystal, are presented.
\end{abstract}

\section{Introduction}

Planar channeling [1] occurs when hadron beams' particles are trapped into the potential well generated by lattice structures with high crystalline order. For beam manipulations at the energy scale of interest for this paper, dislocation densities of the order of one per squared centimeter are typically required. In this case, the particle motion is governed by the crystal potential averaged along the crystalline planes [2]. Channeling is possible in a bent crystal if its bending radius is larger than the critical radius [3]. In this case, channeled particles can be deflected by the same angle of the crystal bending,

\footnotetext{
a e-mail: Stefano.Redaelli@ cern.ch (corresponding author)
}

producing a non-zero deflection of their trajectory. This technique enables achieving fields equivalent to hundreds of tesla in volumes of a few cubic millimeters. The bending angle experienced by hadrons in a bent crystal is expected to be the same for different energies and charges of the incoming particles. However, ionization losses and nuclear interaction cross sections are considerably different for heavy ions and for protons. The possible usage of bent crystal for beam manipulations of high-energy heavy-ion beams calls for a solid experimental demonstration that channeling of circulatingbeam halos can be achieved with adequate efficiencies. The beam measurements presented here surpass by a factor 16 the previous state-of-the-art in terms of beam energies [4].

Crystal channeling with bent crystals can find various applications to high-energy accelerators. At CERN, this is being studied as a possible path to improve the halo collimation of heavy ion beams at the Large Hadron Collider (LHC). In a "crystal collimation system", bent crystals would replace primary collimators and coherently steer halo particles onto a single absorber per beam and per plane (horizontal and vertical). The demonstration of planar channeling at the energy scales of interest for the LHC is essential before relying on this technology for the future operations with heavy ions.

Previous relevant results with heavy ions were obtained at the BNL Relativistic Heavy Ion Collider (RHIC) [5], where channeling of $100 \mathrm{Z} \mathrm{GeV}$ gold beams was achieved; however, these results are not directly usable to conclude on the LHC beam collimation applications. More recently, channeling was observed with lead-ion beams at the CERN Super Proton Synchrotron (SPS) [6] at $270 \mathrm{Z} \mathrm{GeV}$, confirming previous measurements at the SPS [4], and indicated promising results in terms of mitigation of dispersive losses downstream of the crystals. On the other hand, a direct comparison to the 
LHC conditions and a demonstration of channeling in the relevant beam energy regimes still needed to be addressed.

In order to study the channeling at the hadron-beam energies relevant for the LHC, and to obtain a direct comparison to the performance of the present LHC collimation system [7], a crystal collimation test stand was installed in 2015 in the betatron collimation insertion [8]. It consists of two crystals, with length of $4 \mathrm{~mm}$ along the beam direction and bent to a target design angle of $50 \mu \mathrm{rad}$, that enable horizontal and vertical beam collimation tests [9]. ${ }^{1}$ Standard LHC collimators, which are part of the LHC betatron cleaning system and are located downstream of the crystals, are used to intercept and probe the channeled halo particles. The setup was tested for the first time at $6.5 \mathrm{Z} \mathrm{Tev}$ with lead ion beams in 2016, following successful tests at the injection energy of $450 \mathrm{Z} \mathrm{GeV}$ that started in 2015. In these tests, fully-stripped $\mathrm{Pb}$ nuclei with $Z=82$ and $A=208$ were used. In this paper, the results of these measurements, which demonstrate the first observation of channeling of heavy-ion beams in the multi-TeV energy regime, are presented. Results obtained with proton beams are reported in [10].

After a brief review of the crystal layouts deployed in the LHC collimation cleaning area, the experimental procedures to demonstrate the onset of channeling are discussed. The main results are then presented, showing a selection of beam measurements and conclusive results that demonstrate channeling of lead-ion beams at $450 \mathrm{Z} \mathrm{GeV}$ and $6.5 \mathrm{Z} \mathrm{TeV}$. Finally, some conclusions are drawn.

\section{Layout of LHC crystal setup}

The crystal collimation test stand was installed in 2015 in the collimation betatron cleaning insertion, referred to as IR7 [7]. This is a warm straight section, about $500 \mathrm{~m}$ long, that houses a three-stage collimation system based on primary (TCP) and secondary (TCSG) collimators and shower absorber (TCLA) collimators for the betatron halo cleaning [8]. The TCP and TCSG collimators have jaws made of a carbon fiber composite (CFC), $60 \mathrm{~cm}$ and $1 \mathrm{~m}$ long, respectively. The TCLAs are made of $1 \mathrm{~m}$-long jaws with active parts built with a heavy tungsten alloy. The crystals are mounted on an assembly that we refer to as "crystal primary collimator" (TCPC). It contains a high-precision goniometer with sub- $\mu$ rad accuracy [11] that is mounted on a special tank with an "O" shaped pipe, which can hide the crystal during the standard operational activity of the accelerator. This design was conceived to minimize the impedance of the device for high-intensity

\footnotetext{
The value of $50 \mu \mathrm{rad}$ is the target bending angle for optimum collimation performance that was however not achieved by the crystal manufacturers for the first installation, as shown in this paper.
}
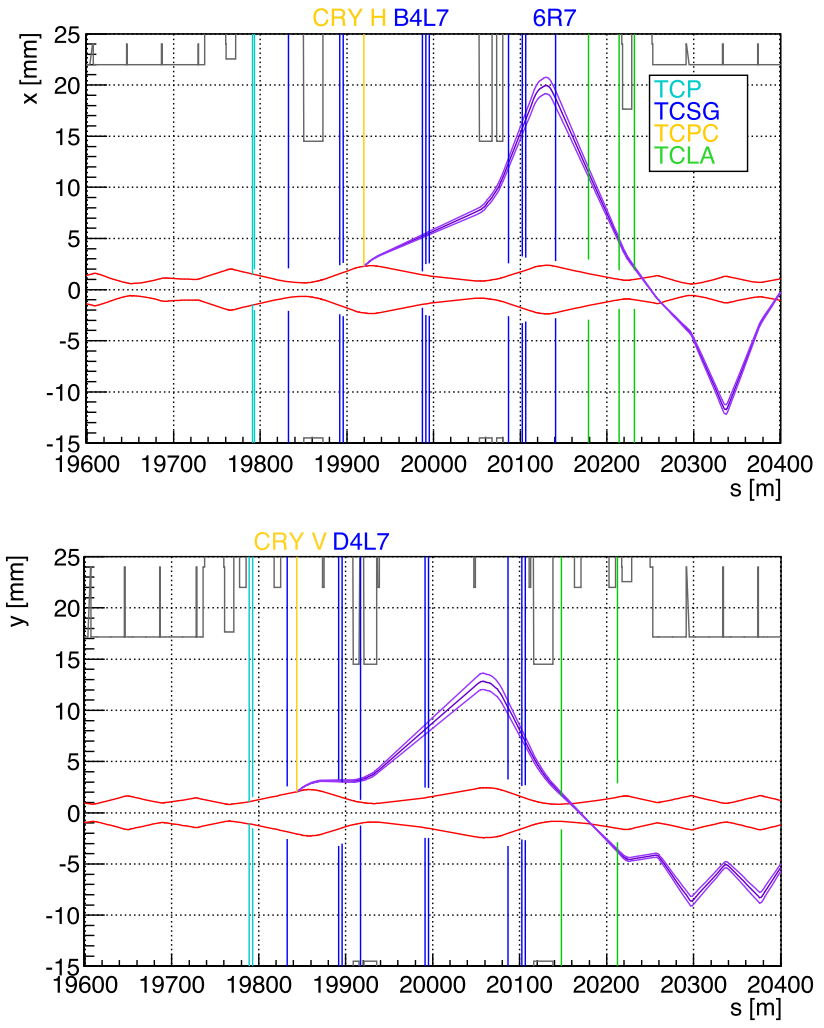

Fig. 1 Trajectory of channeled halo particles (purple lines) for the horizontal (top graph) and vertical (bottom) crystals for the beam tests configurations at $6.5 \mathrm{Z} \mathrm{TeV}$. The envelope of the circulating beam at the amplitude of the crystal settings (5.5 $\sigma$ in this case) is also given (red lines). Vertical lines indicate the aperture of the IR7 collimators in the plane of interest and black lines show the (fixed) aperture other machine elements

proton operations, when the goniometer is in its parking position.

The layout of the LHC crystal setup is described in detail in [9]. It was optimized for the operation with proton beams, but it is fully adequate for ion beam tests as well. A horizontally-bending crystal and a vertically-bending crystal are installed at the longitudinal positions $s=19,919.24 \mathrm{~m}$ and $s=19,843.82 \mathrm{~m}$, respectively, as shown in Fig. 1 (following the notation to measure the distance from the LHC's first interaction point, for the clock-wise "Beam 1", at $s=0$ ). The optics parameters at the crystal locations are given in Table 1.

This crystal collimation test stand relies on standard collimators to intercept the channeled beams. Specifically, the secondary collimators used as absorbers for the channeled halo have layout names TCSG.B4L7.B1, TCSG.6R7.B1 (horizontal) and TCSG.D4L7.B1 (vertical). They are also indicated in Fig. 1 and Table 1. Note that in most experimental tests, the TCLA collimator further downstream were kept closed at their nominal positions to minimize the leakage of channeled halo during beam tests as well as the leakage 
Table 1 Installation position, collimation plane and main optics parameters for the crystal devices (TCPC) and secondary collimators (TCSG) used as absorbers for the channeled halo. Nominal optics parameters are used. Longitudinal installation positions are measured from the first LHC collision point. The position of the middle point of the collimation insertion, IP7, is at $19994.16 \mathrm{~m}$

\begin{tabular}{lllllllllllll}
\hline Name & $s(\mathrm{~m})$ & Collimation plane & Length $(\mathrm{mm})$ & Mat. & Bending planes & $\beta_{x}(\mathrm{~m})$ & $\beta_{y}(\mathrm{~m})$ & $\alpha_{x}(\mathrm{rad})$ & $\alpha_{y}(\mathrm{rad})$ & $D_{x}(\mathrm{~m})$ & $D_{y}(\mathrm{~m})$ \\
\hline TCPCV.A6L7.B1 & $19,843.62$ & Ver. & 4 & $\mathrm{Si}$ & 111 & 30.5 & 281.1 & 0.24 & -2.63 & 0.08 & -0.65 \\
TCPCH.A4L7.B1 & $19,919.49$ & Hor. & 4 & $\mathrm{Si}$ & 110 & 342.1 & 64.9 & -2.05 & 0.84 & -0.68 & -0.27 \\
TCSG.D4L7.B1 & $19,917.24$ & Ver. & 1000 & CFC - & 333.0 & 68.9 & -2.01 & 0.90 & -0.67 & -0.29 \\
TCSG.B4L7.B1 & $19,987.16$ & Hor. & 1000 & CFC - & 139.8 & 131.0 & 1.43 & -1.25 & -0.53 & -0.05 \\
TCSG.6R7.B1 & $20,141.02$ & Hor. & 1000 & CFC - & 335.8 & 47.0 & 2.83 & 0.02 & -0.30 & 0.21 \\
\hline
\end{tabular}

Table 2 Setting in units of beam $\sigma=\sqrt{\beta \epsilon}$, computed for a normalized emittance $\epsilon^{*}=3.5 \mu \mathrm{m}$, at the injection ( $450 \mathrm{Z} \mathrm{GeV}$, "INJ") and flattop (6.5Z Tev, "FT") energies for the relevant IR7 devices used in the channeling experiment: crystals (TCPC), primary collimators (TCP), secondary collimators (TCSG), and shower absorbers (TCLA). Settings of TCSGs upstream and downstream of the crystals are given separately

\begin{tabular}{llllll}
\hline \multirow{2}{*}{ Collimator } & \multicolumn{2}{l}{ Setting $[\sigma]$} & & \multicolumn{2}{l}{ Standard system } \\
\cline { 2 - 3 } \cline { 5 - 6 } & \multicolumn{2}{l}{ Crystal collimation test } & & INJ & FT \\
\cline { 2 - 3 } & INJ & FT & & Out & Out \\
TCPC & 5.7 & 5.5 & & 5.7 & 5.5 \\
TCP & Out & Out & & 6.7 & 7.5 \\
TCSG (upstr.) & Out & Out & & 6.7 & 7.5 \\
TCSG (downst.) & 6.7 & 7.5 & & 10.0 & 11.0 \\
TCLA & 10.0 & 11.0 & & &
\end{tabular}

of showers generated at the TCSG absorbers. A summary of IR7 collimator settings deployed during the crystal test are given in Table 2 and compared to those of the standard collimation system, both at injection (INJ) and flat-top (FT) energies. Note that all the TCP and the TCSG collimators upstream of the crystals are taken out at parking positions during the crystal collimation experiment.

The crystals for this experiment were provided by the UA9 collaboration. Two different silicon crystal technologies were adopted. A strip (ST) crystal [12] produced by Istituto Nazionale di Fisica Nucleare (INFN), Ferrara section, [13] is installed as the horizontal TCPC and a quasi-mosaic (QM) crystal produced by St. Petersburg Nuclear Physics Institute (PNPI) [14] as the vertical one. The ST crystal makes use of the (110) planes that are equidistant while the QM crystal uses the (111) planes, with a ratio of 1:3 for the distances between subsequent planes. The two crystals also use different technologies for the holder that produces the desired geometrical bending: the ST holder uses anti-clastic forces, and the QM one uses the quasi-mosaic effect to bent the desired planes. In both cases, the crystal holder confers the primary deformation that produces the bending of the crystalline planes used to deflect charged particles. The design specification for both crystals was a bending angle of $50 \mu \mathrm{rad}$ and $4 \mathrm{~mm}$ of length [9].

Both crystals were specified to have torsion - defined in units of $\mu \mathrm{rad} / \mathrm{mm}$ as the variation of the crystalline plane orientation along the direction perpendicular to the bending plane - below $1 \mu \mathrm{rad} / \mathrm{mm}$. For the beam conditions at the LHC, with RMS transverse beam sizes below $200 \mu \mathrm{m}$ in the plane orthogonal to the bending plane, effects from torsion are expected to be negligible. For both crystal technologies, one would need torsion values in excess of 15-20 $\mu \mathrm{rad} / \mathrm{mm}$ to have particles at $1 \sigma$ outside the channeling acceptance. Such values seem excluded for the small crystals used for the LHC, based on previous experience. Also note, that at top energy, typical half aperture of the primary collimator cut are below $1.5 \mathrm{~mm}$ for both planes, so large transverse offsets of particles impinging on the crystals are excluded.

The two crystals differ instead for the so called miscut, which is defined as the angle of the crystalline planes with respect to the surface facing the beam. The miscut should be minimised in order to avoid that particles close to the surface experience deflection angles different than the design bending angle. The ST crystal is claimed by the manufacturer to achieve a miscut below $10 \mu \mathrm{rad}$ while the QM crystal is at levels of about $40 \mu \mathrm{rad}$. The ST crystals are built with techniques that promise surface roughness values down to levels of a few atomic layers, at least locally (see for example [15]). No such measurements are available for the QM crystals, although an extensive literature on tests at lower energies than the LHC case discussed in this paper, indicates no significant performance differences between the two crystal types (see for example [6]).

\section{Experimental procedures and beam conditions}

The demonstration of channeling of circulating beam halos relies on two key measurements that are schematically illustrated in Fig. 2:

(1) Crystal angular scans, with the crystal transversally placed into the halo as primary aperture restriction, con- 


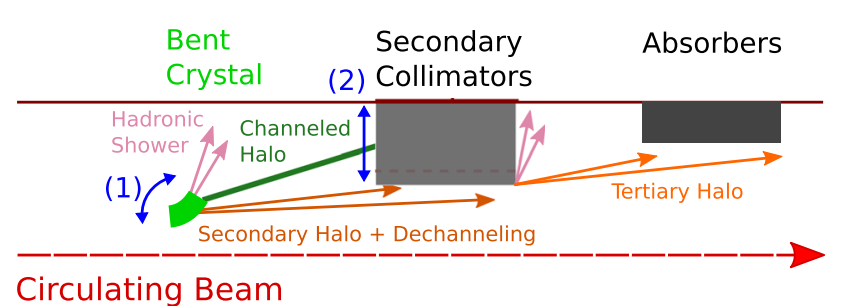

Fig. 2 Illustrative view of the crystal angular scan (1) and of the collimator linear scan (2) that are used to demonstrate experimentally the onset of channeling. Angular scans are used to find the optimum channeling orientation when nuclear losses are minimized. Collimator scans probe the distribution of the channeled halo and the de-channeling region

sists in varying its angular orientation. The onset of channeling is identified as a drop of the local losses that occurs when the channeling planes are parallel to the halo trajectory, as in this condition the nuclear interactions are at a minimum.

(2) Linear scans of the downstream collimator absorber are performed when the crystal is at the optimum angular orientation. The jaw intercepts first the channeled halo and then is moved further in, across the "empty" space, until it reaches the circulating beam core. This produces a sharp loss spike upon reaching the primary beam halo.

Both measurements produce very characteristic distributions of losses versus crystal angle and collimator position, described in the following, that only occurs if channeling is present. Local losses were measured using the standard Beam Loss Monitoring (BLM) system of the LHC [16] based on ionization chambers. The LHC BLM system is ideal for these measurements because it has a good sensitivity and each monitor is capable to measure losses as small as $10^{-7} \mathrm{~Gy} / \mathrm{s}$. The measurements were performed while inducing controlled losses that were in all conditions well above the BLM noise at the monitors of interest.

The beam tests were performed by using several lowintensity bunches both at injection and at flat-top energy, with the standard machine configuration for ion beams in the 2015 (injection only) and 2016 LHC runs [17]. In standard conditions, the single-beam lifetime at the LHC typically exceeds $100 \mathrm{~h}$ and thus the instantaneous losses - i.e., the flux of particles impinging on the crystals - are too low to perform with sufficient resolution the measurements described above. Therefore, the LHC transverse damper [18] was used to excite the beam transversally to increase, in a controlled manner, loss rates. This is done by adding on selected bunches a gated white-noise excitation, of tunable amplitude, that blows up the beam emittance. This enables one to achieve controlled primary beam losses on crystals and/or collimators. Several low-intensity bunches were accelerated to flat-top energy and selectively excited to perform different sets of measurements.

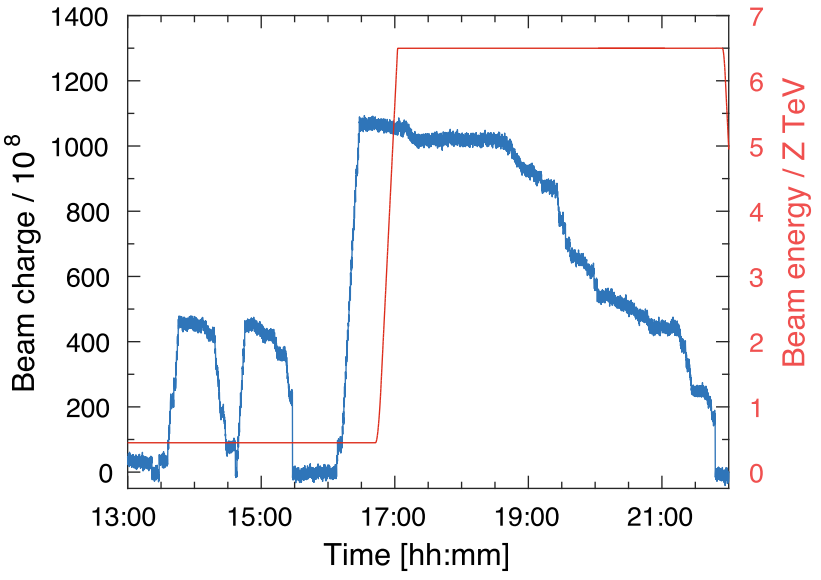

Fig. 3 Beam intensity in charges (blue, left axis) and energy (red, right axis) as a function of time during the beam measurements, performed on Nov. 29th, 2016

The excitation settings were empirically tuned to produce sustained losses during the time needed to complete angular and linear scans (up to several minutes are needed for high-accuracy angular scans). The excitation window was enlarged in time to act on three adjacent bunches. An example of beam current versus time during a beam test at top energy is given in Fig. 3 to illustrate how loss rates were tuned.

During measurements, the crystal acts as a primary collimator and intercepts all primary beam losses. The expected impact parameter of single ions impinging on the crystal defined as the distance from the crystal surface facing the beam at the first interaction - is estimated to be well below $10 \mu \mathrm{m}$ (see for example estimates in [19]). The crystal intercepts only a small fraction of the phase-space ellipse of the beam distribution and this constrains severely the angular values of impinging particles. At $5.5 \sigma$ and $6.5 \mathrm{Z} \mathrm{TeV}$, impact parameters values above $50 \mu \mathrm{m}$ would be required to cause angles of impinging particles above the acceptance for channeling. Effects from the angular distribution can therefore be neglected in first approximation.

Measurements at top energy presented here were obtained during one single fill that lasted about $4 \mathrm{~h}$, see Fig. 3. An initial setup at $450 \mathrm{Z} \mathrm{GeV}$ allowed to rapidly establish the reference channeling orientations for both crystals and to perform a few key measurements before ramping up the beam made of 18 bunches for the measurements at $6.5 \mathrm{Z} \mathrm{TeV}$. The total beam intensity was of about $10^{11}$ charges. The efficient setup of these tests also profited from previous measurements done with proton beams (see for example [10]) when key hardware preparatory tests could be performed.

\section{Measurement results}

The results of the first precise measurements of crystal angular scans at injection energy are shown in Fig. 4 for the 

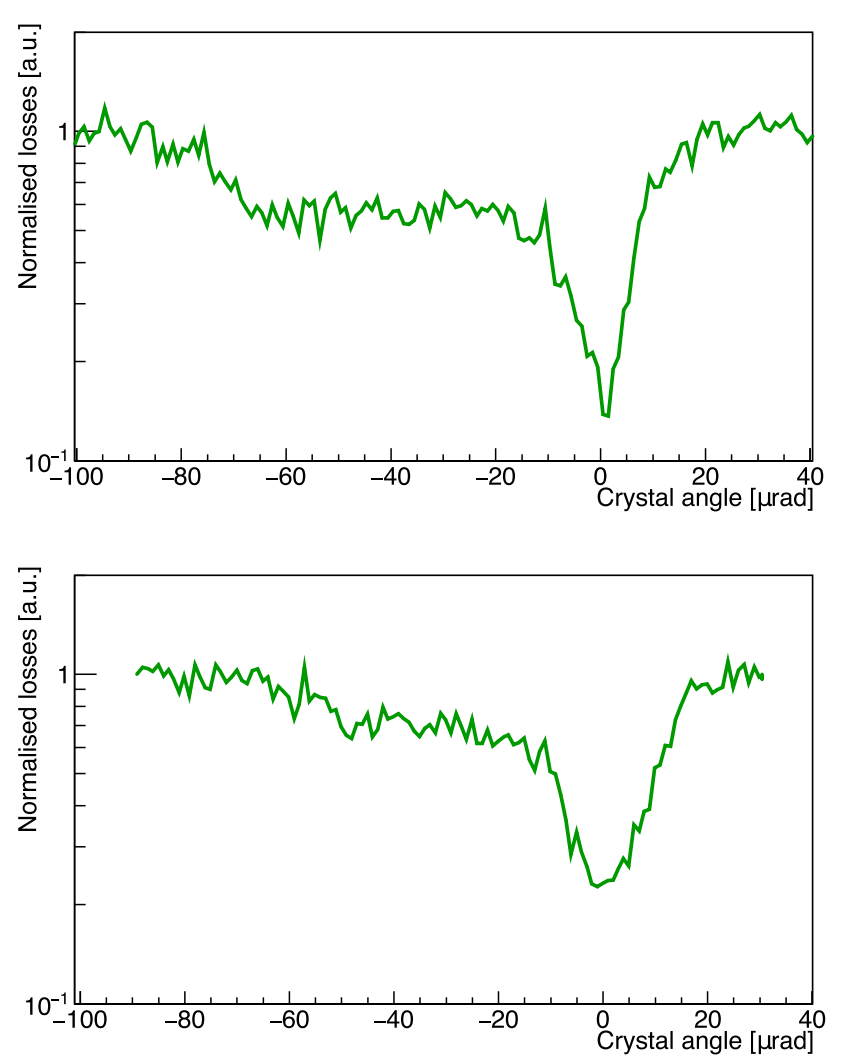

Fig. 4 Local beam losses normalized to the beam flux, and to the BLM signal in amorphous-like orientations, as a function of the crystal angle for the horizontal strip (top) and vertical quasi-mosaic (bottom) crystals. Scans were performed at $450 \mathrm{Z} \mathrm{GeV}$

horizontal (ST) and the vertical (QM) crystals, respectively. Beam losses measured at a downstream BLM of each crystal, normalized to the flux of primary beam losses and to the BLM signal recorded well outside the channeling orientation, are given as a function of the crystal angle. This normalization shall give measurement values close to 1 , independently of the rate of primary beam losses, when the crystal is not in channeling. We call this condition "amorphous-like" orientation because in this case no coherent interactions are observed and thus the interaction of the hadron beam with the crystal is equivalent to that of an amorphous silicon block of the same dimensions.

No beam, collimator or machine parameter is changed, other than the crystal orientation, during this measurement. Movements are performed with angular resolution of $0.1 \mu \mathrm{rad}$. The goniometer accuracy is of about $1 \mu \mathrm{rad}$. The rotational velocity is $1 \mu \mathrm{rad} / \mathrm{s}$ at $450 \mathrm{Z} \mathrm{GeV}$ and $0.2 \mu \mathrm{rad} / \mathrm{s}$ at $6.5 \mathrm{Z} \mathrm{TeV}$ where the critical angle is smaller. A decrease in local losses is clearly seen for both crystals at a specific angle. This orientation providing minimum losses, defined as the zero of the abscissa in the graphs, corresponds to the optimal position for channeling. The crystal planes are parallel to the beam envelope at $5.5 \sigma$ (see Table 2). This reduc- tion of losses can only be explained by the onset of coherent channeling that partially suppresses the interactions of halo particles with the material compared to the amorphous-like orientation, since particles are travelling in a relatively empty space between crystalline planes. This characteristic loss profile is a direct demonstration of channeling, which was never established before for lead ion beams at $450 \mathrm{Z} \mathrm{GeV}$.

It is noted that the angular region between about -60 and $-10 \mu \mathrm{rad}$ in the top graph of Fig. 4 exhibits reduced losses compared to the amorphous-like orientations at either extremity of the angular scan. This corresponds to the $\mathrm{vol}$ ume reflection phenomenon where particles impinging on the convex crystal surface are bounced back by the potential of the crystalline planes [20]. In this example, losses are reduced to about $50-60 \%$ of the amorphous-like losses.

The observations of channeling were also made in similar measurements carried out at $6.5 \mathrm{Z} \mathrm{TeV}$, which are reported in Fig. 5. Channeling is also very clear in this case although the BLM signal is more noisy. This is because losing the beam at top energy entails long procedures to re-establish the same conditions, thus lower loss rates were induced than what could be afforded at injection. The width of the region of around the angle for minimum losses, is narrower than at injection energy because the critical angle for channeling is 3.8 times smaller, i.e. only about $2.5 \mu \mathrm{rad}$. These measurements also demonstrate that the hardware developed for the LHC is adequate for channeling tests in the multi$\mathrm{TeV}$ regime, confirming the excellent performance that was achieved in proton beam tests.

A loss-reduction factor can be measured between the crystal amorphous-like and channeling orientations, using BLMs immediately downstream of the crystals. The loss reductions are presented in Table 3, for both crystals and both beam energies, using the complete set of available data (see $[21,22]$ for a complete overview of available experimental data). It is noted that these factors are achieved for the collimator settings in Table 2. The dependence of the local reduction factor on collimator settings around the ring are subject of other ongoing studies, see for example the results at the SPS [23]. Specifically, this reduction factors are not directly related to the collimation cleaning performance that, for a system complex like the LHC collimation system, depends on the settings of all collimators around the ring.

Figure 6 shows the dependence of the local losses at the TCSG absorber as a function of the jaw positions. This measurement was recorded for the vertical case, with the crystal set at the position for optimum channeling. Similar measurements are available for the other crystal, at both energies, showing qualitatively a similar behaviour [23]. These data can be used to calculate the crystal's bending angle using the beam transport matrix formalism. The crystal kick in $\mu \mathrm{rad}$, $\theta_{\text {cry }}$, e.g. in the horizontal plane, can be expressed as a function of the transverse position of the channeled halo at the 


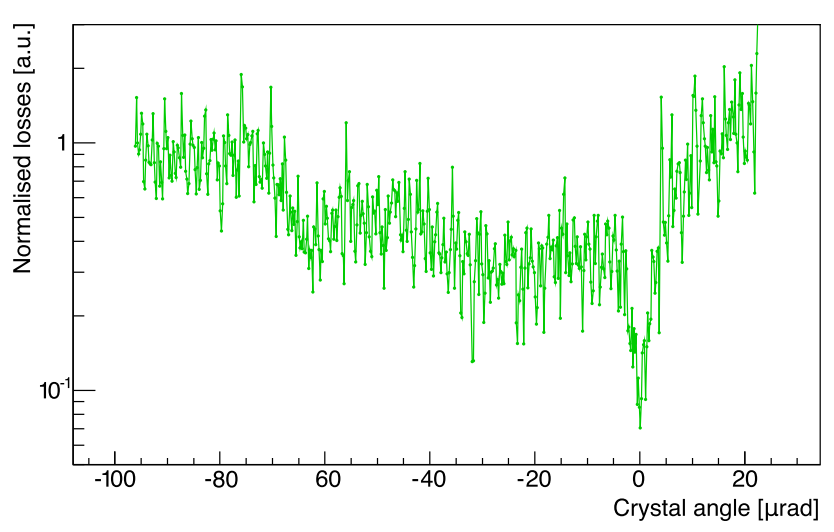

Fig. 5 Local beam losses normalized to the beam flux, and to the BLM signal in amorphous-like orientations, as a function of the crystal angle for the horizontal crystal. The scan was performed at $6.5 \mathrm{Z} \mathrm{TeV}$ with the horizontal crystal on Beam 1

collimator absorber as:

$\theta_{\text {cry }}=\frac{x_{\text {coll }}-\sqrt{\frac{\beta_{\text {coll }}}{\beta_{\text {cry }}}}\left(\cos \varphi+\alpha_{\text {cry }} \sin \varphi\right) x_{\text {cry }}}{\sqrt{\beta_{\text {cry }} \beta_{\text {coll }}} \sin \varphi}-x_{\text {cry }}^{\prime}$,

where $\varphi$ is the betatron phase advance between crystal and absorber, $x$ and $x^{\prime}$ are the transverse coordinates and $\alpha$ is the Twiss parameter (see Table 1). The labels "cry" and "coll" indicate the crystal and the collimator, respectively.

The channeled beam is intercepted when the jaw is moved from its outermost position closer to the circulating beams. This is indicated by the rise of losses at position larger than about $40 \mu \mathrm{rad}$ in Fig. 6. A fit of this profile with an error function can be used to evaluate the position of the channeled beam at the collimator and, through Eq. (1), the crystal bending angle. This amounts to $39.8 \pm 0.4 \mu \mathrm{rad}$ in this case. ${ }^{2}$

At positions even closer to the circulating beam, there is a region of nearly flat losses where only dechanneled particles, or particles that are interacting in other ways with the crystal, are intercepted in addition to the channeled halo. This region continues until the jaw becomes closer to the beam than the crystal itself. It intercepts in this case the primary halo. This breaks the channeling mechanism and produces a sharp loss spike at the TCSG, which is now the primary scatterer. The summary of bending angle measurements obtained with this method is summarized for both crystals in Table 3, were the average angles from the different available measurements are reported. Both crystals differ by about $20 \%$ from the design angle of $50 \mu \mathrm{rad}$.

2 The fit with the error function is applied in the range where the first rise of losses versus collimator position is observed, i.e. in the range above $2.6 \mathrm{~mm}$ in the abscissa of Fig. 6.
Table 3 Reduction factors of local losses in channeling and crystal bending angles computed from different data sets. The average values are presented with the error evaluated as the RMS of available data sets. Bending angles are computed as averages of measurements at injection and top energy

\begin{tabular}{llll}
\hline Crystal & \multicolumn{2}{l}{ Reduction factor } & Bending angle $(\mu \mathrm{rad})$ \\
\cline { 2 - 3 } & Injection & Flat top & \\
\hline B1-H & $6.1 \pm 0.5$ & $8.3 \pm 1.2$ & $62.6 \pm 0.4$ \\
B1-V & $5.6 \pm 0.8$ & $6.2 \pm 2.3$ & $40.0 \pm 0.3$ \\
\hline
\end{tabular}

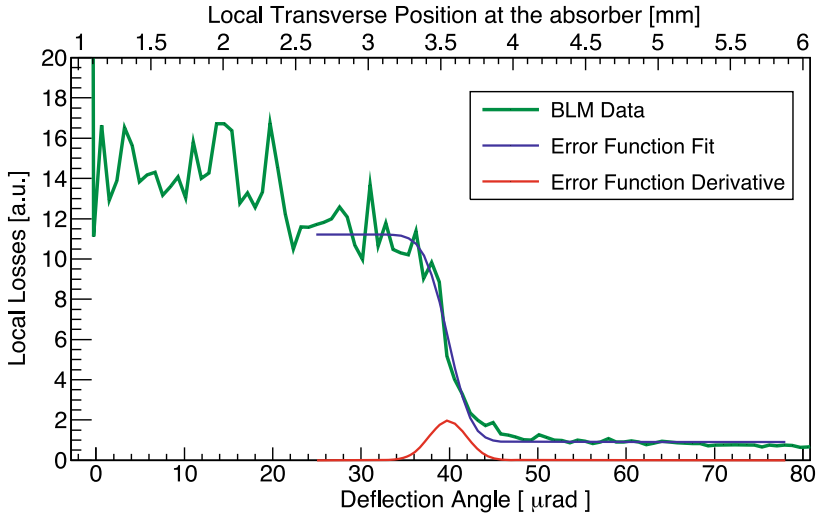

Fig. 6 Beam losses as a function of jaw position and the equivalent deflection at collimator position, recorded at the TCSG.D4L7.B1 during a linear scan with vertical crystal in channeling orientation. The collimator used as absorber has an active jaw of $1 \mathrm{~m}$ of CFC (see Table 1) that does not stop all the energy carried by the channeled halo, but is adequate for the measurement of deflection angle

\section{Conclusions}

Channeling of fully stripped $\mathrm{Pb}$ ion beams $(Z=82$, $A=208$ ) was observed for the first time at the CERN Large Hadron Collider at the unprecedented beam energies of $450 \mathrm{Z} \mathrm{GeV}$ and $6.5 \mathrm{Z} \mathrm{TeV}$, in dedicated machine experiments carried out in 2015 and 2016. Measurements were performed with bent crystals exploiting quasi-mosaic or anti-clastic deformations, for vertical and horizontal halochanneling studies, respectively, using the crystal collimation setup installed in the LHC. Channeling is demonstrated through measurements of reduction of beam losses immediately downstream of the crystals during angular scans as well as through linear scrapings of the channeled halo. At $6.5 \mathrm{Z} \mathrm{TeV}$, measurements indicated a reduction factor of local losses of $6.2 \pm 2.3$ and $8.3 \pm 1.2$ for the quasi-mosaic and the strip crystals, respectively, between channeling and amorphous-like orientations. The measurement uncertainty is high because of the limited available statistics that at this stage does not allow to firmly conclude on systematic difference between the two crystal types. It is important to recall that this local loss reduction factor is not directly related to the collimation cleaning performance for a machine as complex 
as the LHC, where details settings of many other collimators around the ring must also be taken into account.

Measurements of the separation between channeled halo and primary beam indicate bending angles of $40.0 \pm 0.3 \mu \mathrm{rad}$ and $62.6 \pm 0.4 \mu \mathrm{rad}$ for vertical and horizontal crystals, respectively. This is consistent with the results of measurements carried out with proton beams and indicates errors in the achieved crystal bending with respect to the target value of $50.0 \mu \mathrm{rad}$, which will have to be improved for future installations. These results provide an important step towards the implementation of crystal collimation for ion beams at present and future multi-TeV hadron colliders. On the other hand, the understanding of differences between the two crystals is under further investigations, in particular the fact that the strip crystal solution using the plane 110 provides better reduction despite of the larger bending angle.

Acknowledgements The authors would like to acknowledge various groups at CERN, whose members have contributed to these tests, in particular the LHC collimation team, the Operations group and the Accelerator and Beam Physics group of the Beams Department, the Survey, Mechatronics and Measurements group and the Sources, Targets and Interactions group of the Engineering Department. This work is supported by the HL-LHC Project. Special acknowledgements go to the UA9 Collaboration, composed of participants from CERN, IHEP, Imperial College London, INFN, JINR, LAL and PNPI. The crystals that were installed in the LHC and used for this publication were fabricated by PNPI and Università of, and INFN Section, Ferrara. In addition, G. Arduini (CERN), M. Giovannozzi (CERN), and A. Taratin (JINR, for the UA9 Editorial Board) are acknowledged for the careful reading of the manuscript. Finally, G. Cavoto (INFN) is warmly thanked by one of the authors (R. Rossi).

Data Availability Statement This manuscript has no associated data or the data will not be deposited. [Authors' comment: All the relevant data from measurements at the LHC are reported in the paper and the analysis used to produce the results is described.]

Open Access This article is licensed under a Creative Commons Attribution 4.0 International License, which permits use, sharing, adaptation, distribution and reproduction in any medium or format, as long as you give appropriate credit to the original author(s) and the source, provide a link to the Creative Commons licence, and indicate if changes were made. The images or other third party material in this article are included in the article's Creative Commons licence, unless indicated otherwise in a credit line to the material. If material is not included in the article's Creative Commons licence and your intended use is not permitted by statutory regulation or exceeds the permitted use, you will need to obtain permission directly from the copyright holder. To view a copy of this licence, visit http://creativecomm ons.org/licenses/by/4.0/.

Funded by SCOAP ${ }^{3}$.

\section{References}

1. A.M. Taratin, Particle channeling in bent crystal. Phys. Part. Nucl. 29, 437-462 (1965)

2. J. Lindhard, K. Dan. Vidensk. Selsk. Mat. Fys. Medd. 34(14) (1965)

3. E.N. Tsyganov, Preprint TM-682 (Fermilab, Batavia, 1976)
4. G. Arduini et al., Deflection and extraction of $\mathrm{Pb}$ ions up to 33 TeV/c by a bent silicon crystal. Phys. Rev. Lett. 79, 4182 (1997)

5. R.P. Filler III et al., Results on bent crystal channeling and collimation at the relativistic heavy ion collider. Phys. Rev. Spec. Top. Accel. Beams 9, 013501 (2006)

6. W. Scandale et al., Comparative results on collimation of the SPS beam of protons and $\mathrm{Pb}$ ions with bent crystals. Phys. Lett. B 703(5), 547-551 (2011)

7. O. Brüning et al., LHC design report, vol. I, CERN, Geneva, Switzerland, Rep. CERN-2004-003-V-1 (2004)

8. R.W. Assmann et al., The final collimation system for the LHC, CERN, Geneva, Switzerland, CERN-LHC-Project-Report919 (2006)

9. D. Mirarchi et al., Design and implementation of a crystal collimation test stand at the Large Hadron Collider. EPJ C 77, 424 (2017)

10. W. Scandale et al., Observation of channeling for $6500 \mathrm{GeV} / \mathrm{c}$ protons in the crystal assisted collimation setup for LHC. Phys. Lett. B 758, 129-133 (2016)

11. M. Butcher, A. Giustiniani, R. Losito, A. Masi, Controller design and verification for a rotational piezo-based actuator for accurate positioning applications in noisy environments. In: IECON 2015 41 st Annual Conference of the IEEE Industrial Electronics Society, Yokohama, pp. 003887-003892 (2015). https://doi.org/10.1109/ IECON.2015.7392706

12. A.G. Afonin et al., First results of experiments on high-efficiency single-crystal extraction of protons from the U-70 accelerator. JETP Lett. 67, 781 (1998)

13. G. Germogli et al., Bent silicon strip crystals for high-energy charged particle beam collimation. Nucl. Instrum. Meth. B 402, 308-312 (2017)

14. Y.A. Ivanov et al., Observation of the elastic quasi-mosaicity effect in bent silicon single crystals. JETP Lett. 81, 99-101 (2005)

15. S. Baricordi, V. Guidi, A. Mazzolari, D. Vincenzi, M. Ferroni, Shaping of silicon crystals for channelling experiments through anisotropic chemical etching. J. Phys. D 41(24), 245501 (2008). https://doi.org/10.1088/0022-3727/41/24/245501

16. E.B. Holzer, B. Dehning et al., Beam loss monitoring for LHC machine protection. Phys. Procedia 37, 2055-2062 (2012) (Contribution to: TIPP 2011),

17. J. Jowett et al., The 2015 heavy-ion run of the LHC, in TUPMWO27, IPAC 2016 Proceedings (2016)

18. W. Hofle, Progress in transverse feedbacks and related diagnostics for hadron machine, in FRXCA01, IPAC 2013, Shanghai (2013)

19. R. Bruce et al., Simulations and measurements of beam loss patterns at the CERN Large Hadron Collider. Phys. Rev. ST Accel. Beams 17, 081004 (2014). https://doi.org/10.1103/PhysRevSTAB. 17.081004. arXiv:1409.3123 [physics.acc-ph]

20. Yu.M. Ivanov et al., Volume reflection of a proton beam in a bent crystal. Phys. Rev. Lett. 97, 144801 (2006)

21. R. Rossi et al., Crystal collimation with lead ion beams at injection energy in the LHC. LHC MD Note, CERN-ATS-Note2018-0004-MD (2018). https://cds.cern.ch/record/2303714/files/ CERN-ACC-NOTE-2018-0004.pdf

22. R. Rossi et al., Crystal collimation cleaning measurements with lead ion beams in LHC. LHC MD note, CERN-ACCNOTE-2018-0077 (2018). https://cds.cern.ch/record/2648626/ files/CERN-ACC-NOTE-2018-0077.pdf

23. R. Rossi, Experimental assessment of crystal collimation at the large hadron collider. PhD Thesis, CERN-THESIS-2017-424 (2017) 